\title{
Gestión para la reducción del riesgo de desastres en Honduras
}

CIENCIA Y TECNOLOGÍA (CT) Entrevista al Profesor Nabil Kawas, Coordinador y catedrático de la Maestría en Gestión de Riesgos de la Facultad de Ciencias, actualmente se desempeña como Director del Instituto Hondureño de Ciencias de la Tierra de la UNAH. Ha sido profesor de varios cursos en Hidrometereologia, Gestión de Riesgos y Recursos Hídricos, Representante de Honduras ante la Organización Meteorológica Mundial, 1986- 1994, miembro del Consejo Ejecutivo de la OM, y Secretario Ejecutivo del Comité Regional centroamericano de Recursos Hidráulicos (1977-1986).

\section{CT/ ¿Cómo está la gestión para la reducción del riesgo de desastres en el país?}

NKI La gestión del Riesgo a desastres en el país se inició por causa del Huracán Mitch, que despertó el interés de las autoridades superiores así como a los tomadores de decisión política y de la sociedad civil. Aunque anteriormente habíamos tenido situaciones similares como el Fifí 1974, Gilberto 1989 y otros, el nivel político y privado no creía en el aumento de la duración e intensidad de estos fenómenos a su paso por la región. Actualmente existe La Ley del SINAGER (Sistema Nacional de Gestión del Riesgo) que busca coordinar todas las acciones de las instituciones miembros del sistema, relacionadas con gestión para la reducción del riesgo de desastres en el país, teniendo como ente coordinador a COPECO.

$\mathrm{CT} /$ ¿Qué instituciones son las que más están trabajando en gestión para la reducción del riesgo de desastres en el país?

NKJ En Honduras existen muchas instituciones que trabajan en este tema. Comenzando en el sector público tenemos a COPECO como el ente coordinador y regulador del sector riesgo, y todas las demás instituciones públicas miembros del sistema de gestión del riesgo que apoyan en temas de prevención, atención, mitigación y rehabilitación ante un desastre. Asimismo está el sector académico en especial el Instituto Hondureño de Ciencias de la Tierra de la Universidad Nacional Autónoma de Honduras que, aunque muy joven, ya ha logrado desarrollar varios estudios técnicos y de investigación en este tema que han contribuido al mejor conocimiento y caracterización de las zonas de mayor exposición a las amenazas en el país. 
El sector de la Sociedad Civil ha estado muy activo en los últimos 13 años (a partir del Mitch), apoyado por la cooperación internacional y países amigos, ejecutando actividades para la reducción del riesgo a desastres (RRD). Y no hay que olvidar al sector privado, que en muchos casos ha estado contribuyendo en estas actividades.

\section{$\mathrm{CT} /$ ¿En qué áreas de la gestión para la reducción del riesgo de desastres se tienen fortalezas en el país y en cuáles debilidades?}

NK/ Definitivamente hemos estado más ocupados en el área de atención y mitigación que en la prevención. Esta última ha sido más débil en especial reflejada por las autoridades superiores del sector público, dado que a la fecha no se ha podido lograr empoderar a los tomadores de decisión de la necesidad urgente de invertir en la prevención con el fin de lograr reducir las pérdidas no sólo de vidas humanas, sino de recursos físicos y socioeconómicos que dejan un endeudamiento mayor del gobierno central cada vez que se presenta un fenómeno hidrometeorológico o de otraíndole.

\section{$\mathrm{CT} /$ ¿Qué investigaciones han contribuido a la gestión para la reducción del riesgo de desastres en el país?}

NKI En Honduras se han desarrollado muchos estudios técnicos y de investigación, pero en muy pocos casos han sido tomados en cuenta para la gestión y preparación de planes a corto, medianoy largo plazo.

Podemos mencionar los estudios de línea base en meteorología, hidrología, geofísica y de cambio climático (todos ellos actualizados muy recientes), basados en información recién recopilada gracias a algunas instituciones públicas que han tenido que generar la información durante muchos años a falta de una institución unificada y no usuaria de la misma que se responsabilice por la producción. Estos registros han servido para desarrollar estos estudios.

\section{$\mathrm{CT} / ¿ Q$ Qué amenazas son las que han causado mayores daños y pérdidas en el país en la última década?}

NKI Las mayores amenazas que causan daños al país son de origen hidrometeorológico, entre ellas podemos mencionar las naturales como los ciclones (Depresiones, Tormentas Tropicales y Huracanes), El Niño, y las geofísicas como los temblores y tsunamis; y en combinación a los naturales los antropogénicos que nos producen sequias, inundaciones, movimientos de laderas (deslizamientos y derrumbes), incluyendo las mareas, marejadas y otros. 


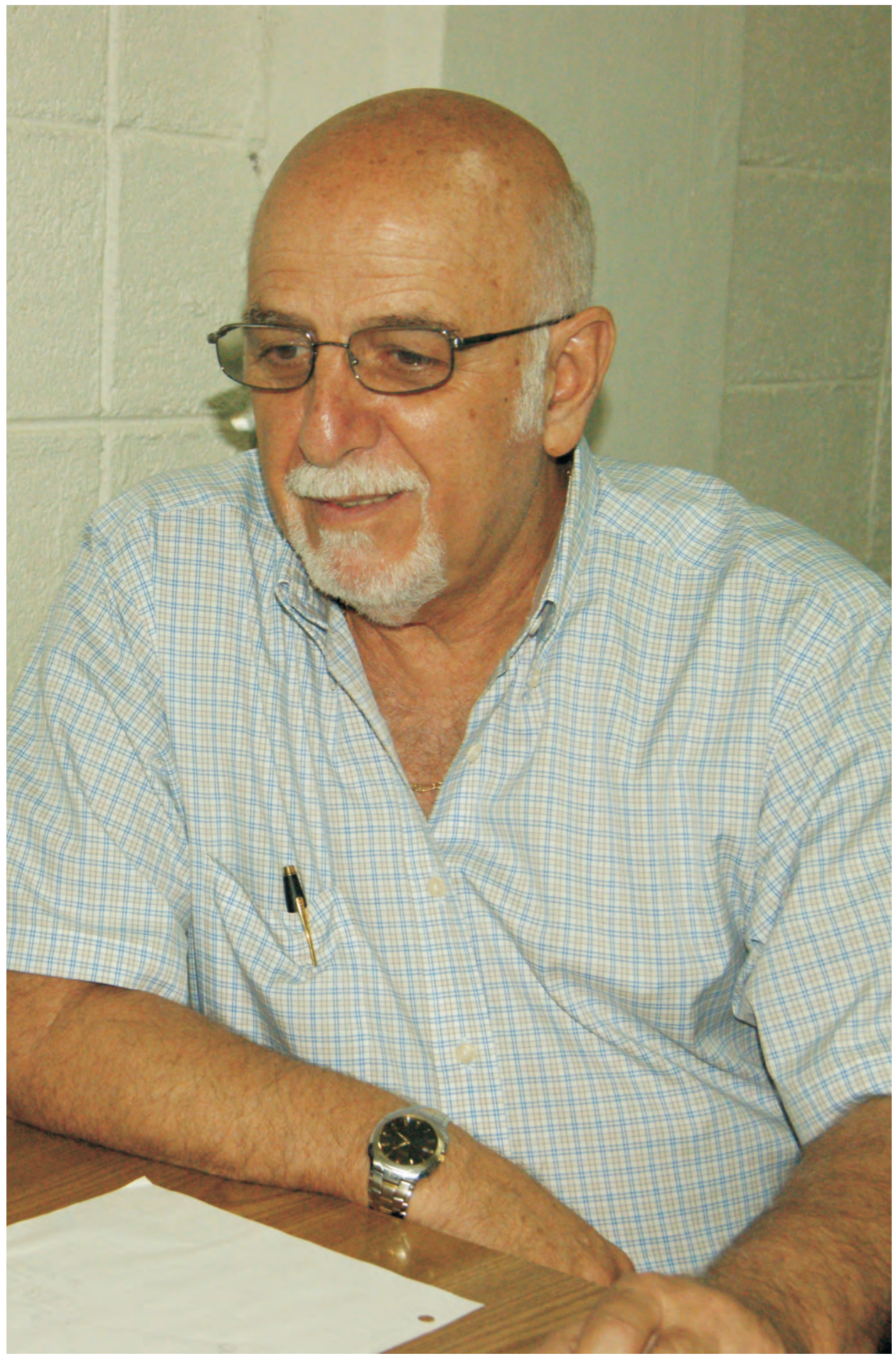

Dirección de Investigación Científica $\longdiv { 2 1 }$ 


\section{$\mathrm{CT} /$ ¿Cómo la amenaza global del cambio climático está afectando al país y cómo lo afectará en el futuro?}

NKI El cambio climático es un fenómeno muy reciente en cuanto a su estudio y análisis, es más un producto de las acciones antropogénicas que naturales, y que ha permitido conocer en alguna medida, de acuerdo a los estudios realizados, cómo estos cambios generan en muy pocos casos beneficios al país, pero que en su mayoría son negativos en los sectores agrícolas en el centro, sur y pacífico, como son los problemas energéticos, contaminación y cambios en la flora y fauna, alejando muchas especies conocidas y atrayendo a otras no muy comunes en la Región Centroamericana y El Caribe.

El futuro del país y de la Región Centroamericana, de acuerdo a los últimos escenarios generados para nuestro país, es que tendremos un aumento de la temperatura en más de $1.5^{\circ} \mathrm{C}$, así como del nivel del mar en nuestras costas, mayormente las del Océano Atlántico y mareas y marejada en el Pacífico con mayor frecuencia. El índice de aridez irá incrementándose paulatinamente y extendiéndose a la mayor parte de nuestro territorio en los próximos 50 años, aumentando nuestra vulnerabilidad ante los efectos de estos cambios y sus consecuencias.

\section{CT/ ¿Qué sector económico es el que ha sido más afectados por desastres de origen natural en el país?}

NKI Los sectores más afectados por desastres producidos por fenómenos de origen natural son varios, pero en primer lugar están las pérdidas de vidas humanas, y a partir de ahí los sectores infraestructura, agrícola y de salud.

\section{CT/ ¿En materia de educación cómo se ha avanzado para contribuir a la reducción del riesgo de desastres?}

NK/ En materia de educación podemos señalar varios aspectos muy importantes. Comenzando a nivel escolar y de media, las autoridades del Ministerio de Educación han aprobado acuerdos gubernamentales que obligan la inserción del tema de gestión de riesgo en toda actividad curricular del sistema educativo, lo cual permite a la población más joven entender y conocer de las amenazas, vulnerabilidades y riesgos a la que está expuesta en su zona y en todo el país por los diferentes fenómenos que se presentan. 
Asimismo en la educación superior. Las autoridades universitarias desde hace 5 años aprobaron el desarrollo de un programa a nivel de maestría en gestión de riesgo que ha venido preparando un buen número de profesionales especialistas en el tema. Actualmente diferentes instituciones del estado y la cooperación internacional y sociedad civil atienden este tipo de emergencias.

\section{CT/ ¿Qué zonas del país son las más vulnerables ante las amenazas de mayor ocurrencia?}

NK/ Por la situación geográfica y ubicación, la región del Caribe. Esta zona está totalmente expuesta a los fenómenos naturales que se presentan anualmente como los ciclones y sismos anteriormente mencionados. En el centro y sur la exposición es mayor a la sequia y aumento de la aridez; y en el resto del territorio nacional, por ser muy montañoso, estamos expuestos a los deslizamientos y derrumbes producto del mal manejo de nuestras cuencas, así como a la intervención del ser humano en las actividades agrícolas no tecnificadas que acompañadas del mal uso del suelo y de las intensas lluvias durante la época lluviosa desarrollan los movimientos de laderas mencionadas anteriormente.

\section{CT/ ¿El país está preparado para hacerle frente a un desastre de grandes proporciones?}

NK/ Definitivamente la respuesta es NO; aunque hemos desarrollado muchas acciones en la mayoría de nuestra población expuesta y se han gastado enormes cantidades de dinero en la atención y rehabilitación. La densidad de población en especial en ciertas aéreas del territorio ha venido aumento, seguimos generando prácticas nocivas y negativas como la acumulación de basura en los lugares de mayor población (creemos que nuestras cuencas con la ayuda del agua limpiaran por nosotros los lechos de los ríos, el azolvamiento de los mismos y otras prácticas mal entendidas). La falta de recursos de diferentes tipos hace que sigamos siendo muy vulnerables a cualquier desastre de gran magnitud. 